\title{
A Case of Candidal Infection of Large Biloma After CT-Guided Liver Biopsy Confirming Autoimmune Hepatitis
}

\author{
Sindhura Kolli ${ }^{1}$, Vahe Shahnazarian ${ }^{2}$, Harini Gurram ${ }^{3}$, Madhavi Reddy ${ }^{2}$, Krishna Gurram ${ }^{4}$ \\ 1. Internal Medicine, New York University (NYU) Langone Hospital, New York, USA 2. Gastroenterology and \\ Hepatology, The Brooklyn Hospital Center, Affiliate of the Icahn School of Medicine Mount Sinai, Brooklyn, USA 3. \\ Internal Medicine, Northwestern University Feinberg School of Medicine, Chicago, USA 4. Gastroenterology and \\ Hepatology, Elmhurst Hospital, Affiliate of Icahn School of Medicine Mount Sinai, Queens, USA
}

Corresponding author: Sindhura Kolli, sindhura.kolli@gmail.com

\begin{abstract}
Bilomas are unexpected post-procedural complications of cholecystectomies, hepatic resections, endoscopic retrograde cholangiopancreatography, and-rarely-liver biopsies. While uncommon, this should remain high on the differential in a patient presenting with sudden postop sepsis even in the absence of significant liver injury. Diagnosis involves coordination between interventional radiology, gastroenterology, and internal medicine. This involves a precise clinical history, invasive radiological techniques, and advanced interventional endoscopic solutions for diagnosis and treatment. This is a case of a biloma that occurred as a consequence of a post-hepatic biopsy and resulted in fungal infection, rather than its more common bacterial counterpart. Due to the unusual circumstances, a more circuitous route to the correct diagnosis and treatment was undertaken.
\end{abstract}

Received 08/26/2020

Review began 10/13/2020 Review ended 10/14/2020 Published 10/20/2020

(c) Copyright 2020

Kolli et al. This is an open access article distributed under the terms of the Creative Commons Attribution License CC-BY 4.0., which permits unrestricted use, distribution, and reproduction in any medium, provided the original author and source are credited.
Categories: Internal Medicine, Gastroenterology, Infectious Disease Keywords: biloma, candida, fungal, autoimmune hepatitis

\section{Introduction}

When faced with sepsis in a patient who recently underwent an invasive procedure near the biliary system, it is essential to exclude biloma even when it is not high on the differential. While mostly asymptomatic and benign, when superimposed with an infection, bilomas can lead to a cascade of complications requiring rapid diagnosis and treatment with either percutaneous drainage or more invasive procedures such as endoscopic retrograde cholangiopancreatography (ERCP) or hepatic resection. This is a case of a biloma that occurred after a liver biopsy. However, instead of becoming infected with more commonly introduced bacteria, the cultures paradoxically demonstrated a fungal infection. Herein, we expand on the known epidemiology, clinical presentation, and the standard methods of diagnosis and treatment of bilomas. Additionally, we use this case to bring light to an uncommon occurrence while proposing a diagnostic and treatment algorithm for intrahepatic bilomas.

\section{Case Presentation}

A 64-year-old male with a medical history of alcohol abuse, chronic pancreatitis, and recent computed tomography (CT)-guided liver biopsy (performed a week prior to admission, confirming autoimmune hepatitis) presented to the emergency department with lethargy, dizziness, anorexia, and intermittent diffuse abdominal pain. Vitals were: $100.1^{\circ} \mathrm{F}$ temperature, heart rate 102 beats per minute, and blood pressure 93/60 mmHg. Abnormal labs included neutrophil-predominant leukocytosis of 22.8 cells/L (4.5 to $11.0 \times 109 / \mathrm{L}$ ), alanine transaminase (ALT) of $111 \mathrm{u} / \mathrm{L}$ (7-56 units/liter), aspartate transaminase (AST) of 134 $\mathrm{u} / \mathrm{L}$ (10-40 units/liters), and total bilirubin of $16 \mathrm{mg} / \mathrm{dL}(0.1-1.2 \mathrm{mg} / \mathrm{dL})$, with $14 \mathrm{mg} / \mathrm{dL}(0.1-0.3 \mathrm{mg} / \mathrm{dL})$ of it direct bilirubin. A CT showed multiple lobulated cystic lesions in the right hepatic lobe communicating with the intrahepatic biliary ducts indicating a biloma, which was later confirmed by magnetic resonance imaging (MRI) with contrast of the abdomen and pelvis (Figure 1). 


\section{Cureus}

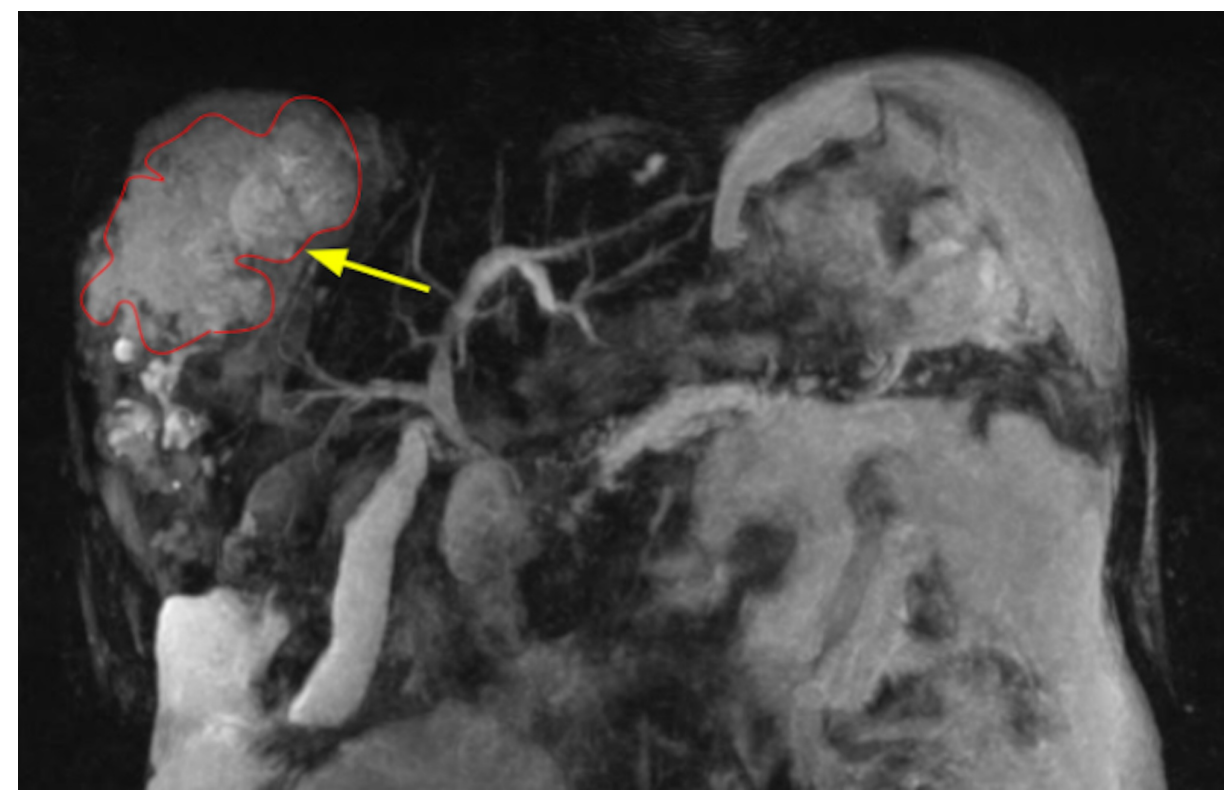

FIGURE 1: Arrow - large biloma collection involving a large portion of the right lobe of the liver; Red line - biloma outline

Piperacillin-tazobactam was empirically started. During the ERCP, fluoroscopic images demonstrated a large biloma in segments seven and eight of the liver (Figure 2A-2C). A 7 French (Fr) x 12 centimeter (cm) stent was placed and the biloma was drained.

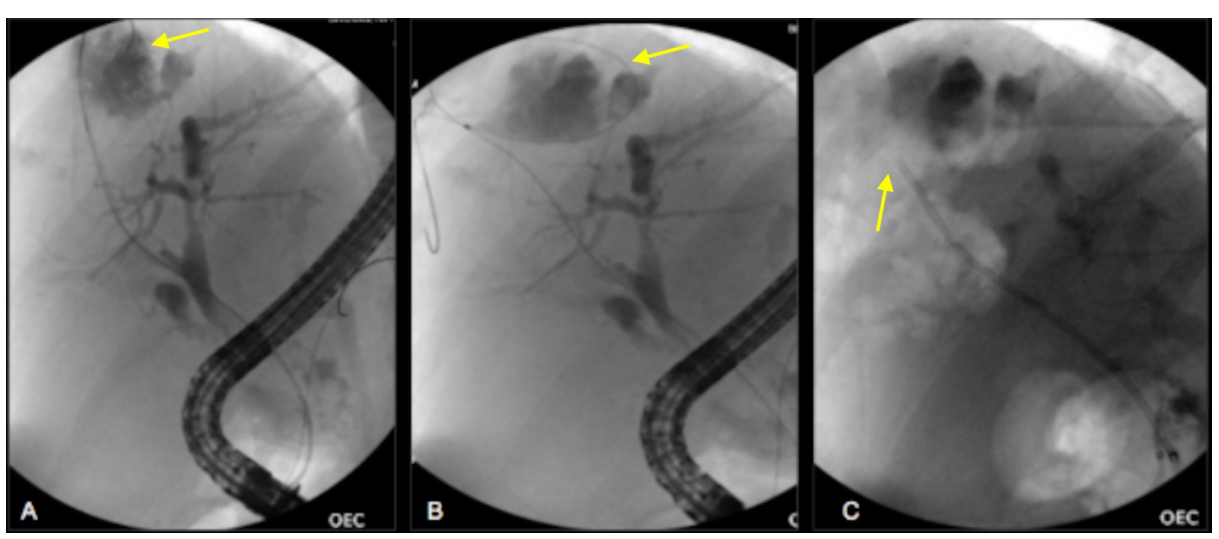

FIGURE 2: Fluoroscopic images in sequential order

2A. Contrast injection into the biloma. 2B. Wire coiled in the large biloma. 2C. Air in the biloma cavity after placement of plastic stent with transpapillary drainage

Culture of the biloma fluid revealed candida and numerous neutrophils, prompting the addition of fluconazole. A postoperative CT of the abdomen showed no change in the size of the biloma (Figure 3). 


\section{Cureus}

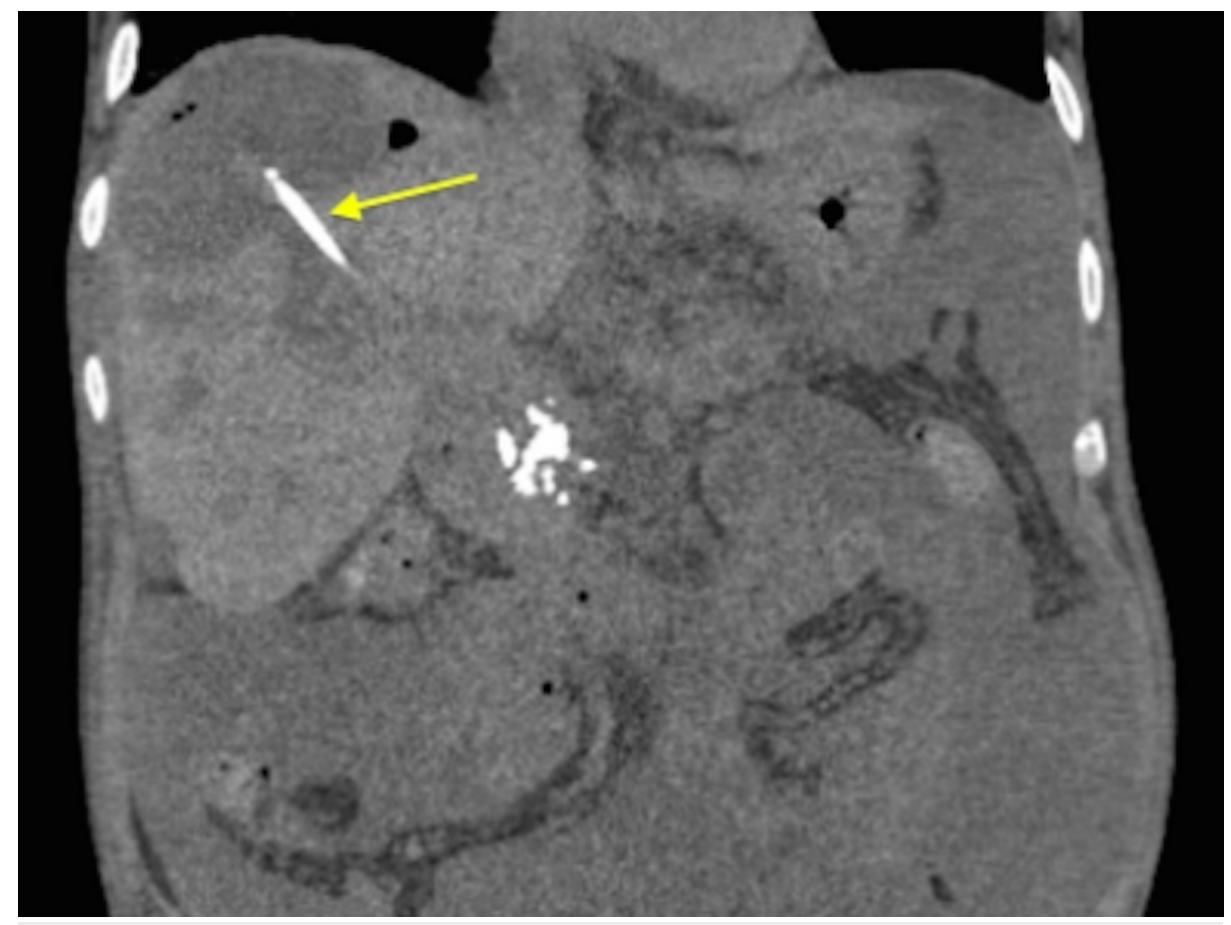

FIGURE 3: Post-procedural CT of the abdomen with an arrow demonstrating the stent within the biloma

The patient left against medical advice but returned with similar complaints two weeks later. While vitals were within normal limits, abnormal labs were similar to the previous admission. A repeat ERCP showed the previous stent was patent, but there was still a large collection and biliary sludge within segments seven and eight of the liver. The previous stent was then replaced with a $10 \mathrm{Fr}$ x $12 \mathrm{~cm}$ (Figure 4). Having

symptomatically improved, the patient again left against medical advice and was lost to follow-up.

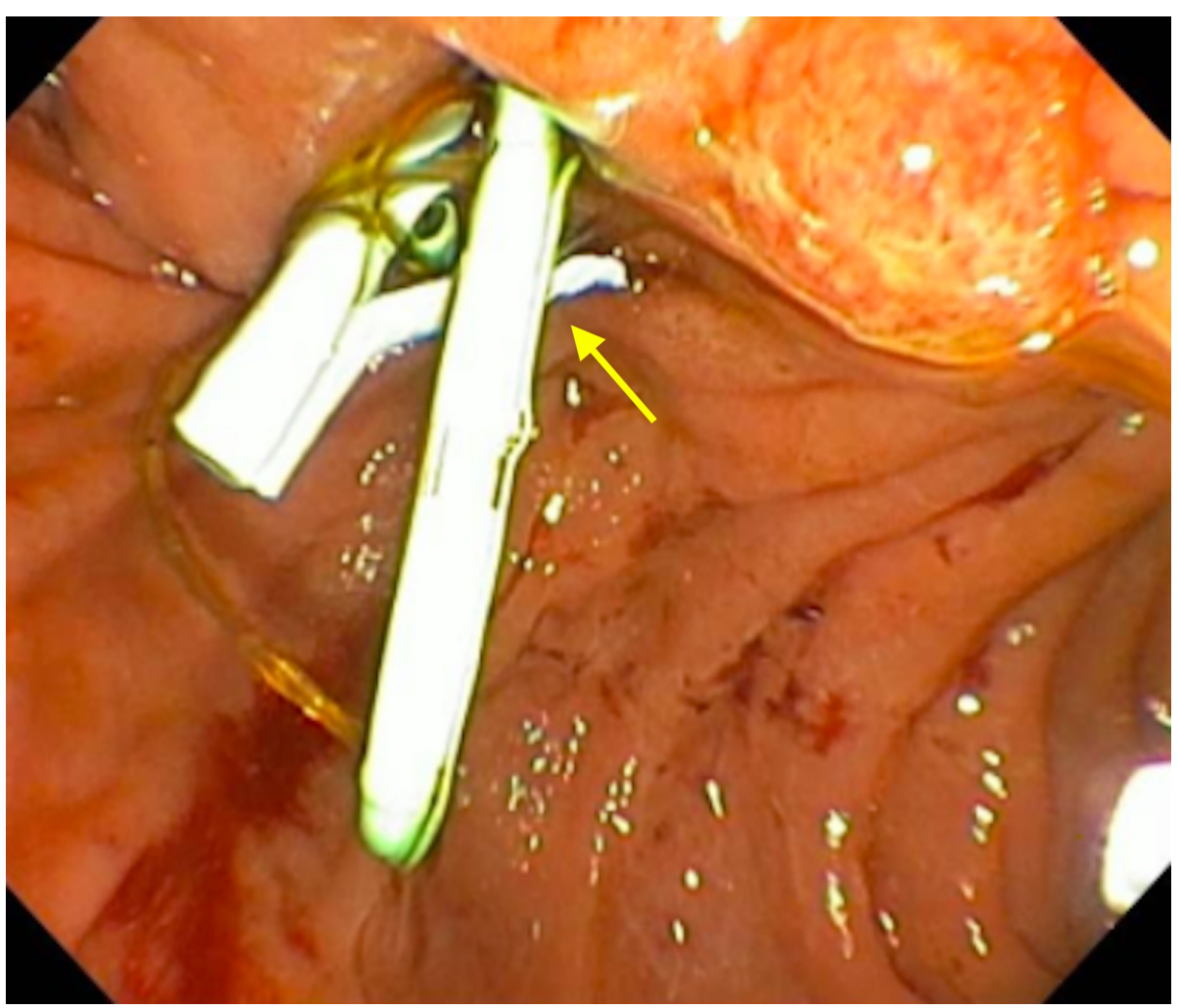

FIGURE 4: Endoscopic image of the stent in place providing biloma 


\section{Cureus}

drainage

\section{Discussion}

Bilomas are collections of bile, either intra- or extrahepatic, with or without a capsule. Their incidence in men and women is equal and occurs in the seventh and eighth decades of life, within a week of the inciting event. They are rare and occur due to disruptions within the biliary system. Disruptions or injuries are often secondary to laparoscopic cholecystectomies, post-Billroth II procedures, poor revision to the cystic duct stump, or puncturing the accessory ducts of Luschka. Other causes include blunt trauma to the abdomen, radiofrequency ablation or resection of hepatocellular carcinoma, and, rarely, spontaneous formation of bilomas secondary to an obstruction or rupture within the biliary system (Table 1) [1-8].

\section{Causes of Biloma}

\section{Most common causes}

Laparoscopic cholecystectomies:

- Poor revision to the cystic duct stump

- Puncturing the accessory ducts of Luschka

Billroth II procedures

Other causes

Liver biopsy

Blunt abdominal trauma

Radiofrequency ablation

Resection of hepatocellular carcinoma

Spontaneous biliary system obstruction or rupture

ERCP

Percutaneous ethanol injection

\section{TABLE 1: Etiology resulting in the formation of bilomas}

ERCP: endoscopic retrograde cholangiopancreatography

Sources: [4-8]

In slow chronic leaks, a reactive process of inflammation and fibrosis creates a capsule supported by adhesions from the surrounding mesentery and omentum to encapsulate the bile. If the bile leak is more acute, resultant peritonitis and sepsis can occur. Secondary infections of the bile within the biloma are another common sequelae. Common organisms include Enterobacteriaceae (43.0\%), Enterococcus (32.2\%), and, less commonly, Candida (9.1\%) [9]. Clinical symptoms range from abdominal fullness, dull right upper quadrant pain to nausea, vomiting, fever, and gram-negative sepsis [1].

Diagnosis begins non-invasively, with an ultrasound (US) showing cysts, anechoic collections, or septated loculations (indicating infection) [10]. Aspiration of the noted collection(s) is needed for confirmation. CT scans are an option but have low specificity and are ineffective in ruling out differentials such as hematomas, seromas, or pseudocysts. The gold standard continues to be a hepatobiliary cholescintigraphy with a Tc-99m-iminodiacetic acid chelate complex as a tracer, which can detect active or sealed leaks and guide treatment. Its limitations in identifying the exact point of leak due to poor demonstration of detailed anatomy can be circumvented with the concurrent use of a hybrid single-photon emission computed tomography (SPECT/CT) or magnetic resonance cholangiopancreatography (MRCP), which provide a more detailed depiction of the origin of leak showing points of connection between the biloma and the biliary system with an exactness of 79\%-85\% [11]. More invasive methods, such as ERCP, can also identify and simultaneously treat bilomas [1-3]. They must be distinguished from differentials such as pseudocysts, seromas, abscess, or hematomas [12].

When asymptomatic and small, bilomas can be monitored with frequent surveillance. Only when they leak 
and create symptoms, or become infected, is intervention indicated. Options for treatment are based upon the patient's overall clinical symptoms. Proceeding directly to an ERCP is possible if adequate mapping of the biloma and intraductal connections is obtained by an MRCP. However, if there are scant or nil biloma duct communications, a percutaneous transhepatic cholangiography (PTC) is done beforehand to illuminate the biliary anatomy before intervention to precisely identify leaks. Then, either a percutaneous transhepatic biliary drainage (PTBD) with an external drain or stricture dilatation or percutaneous drainage are options. An ultrasound (US) or CT afterwards is necessary to confirm that the biloma had drained. If still persistent, an ERCP to decompress, drain, and stent the biloma in combination with a PTC or surgical resection of the liver with biloma are options once the patient is no longer septic (Figure 5) [1-3].

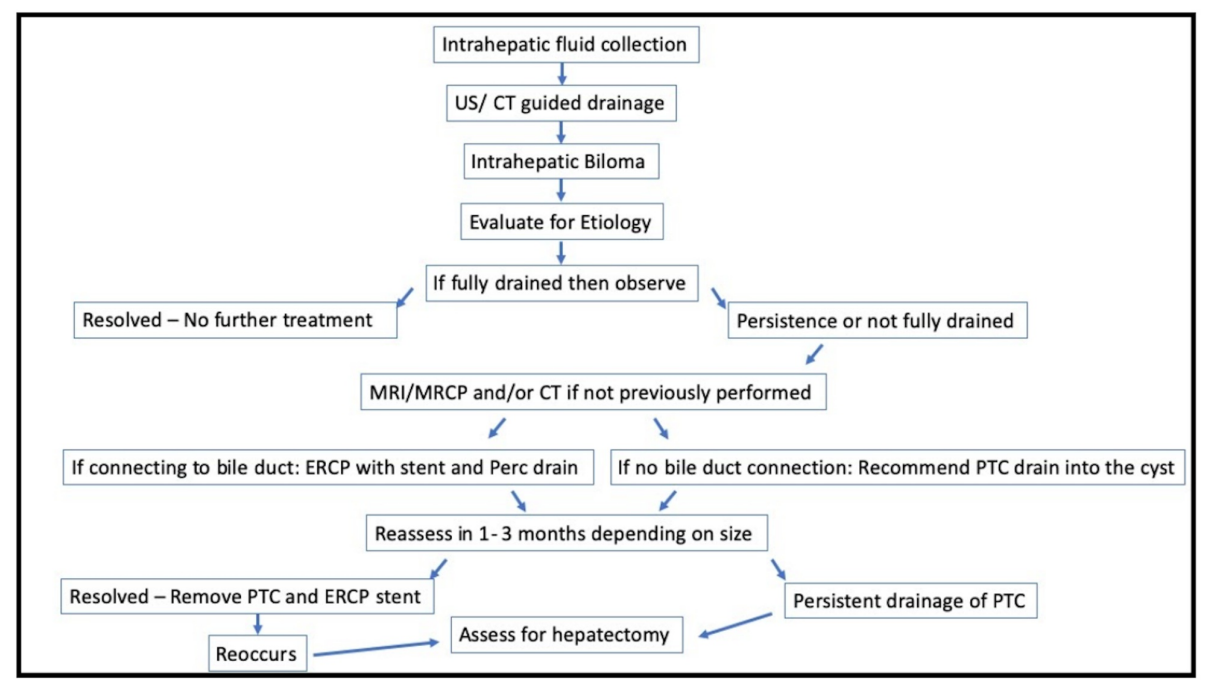

FIGURE 5: Proposed algorithm for an intrahepatic biloma

\section{Conclusions}

When asymptomatic and of small size, bilomas do not need to be disturbed. However, once infected or symptomatic, they require immediate diagnosis and intervention. Mapping of the communication prior to an ERCP decompression and confirmatory imaging postoperatively is ideal. Cultures are recommended instead of empirical antibacterials, given that, in this case, the source was fungal and would have prolonged symptoms without resolution. When bilomas are recurrent, a repeat ERCP with a stent might be necessitated, thus making postoperative imaging key to the treatment course.

\section{Additional Information}

\section{Disclosures}

Human subjects: Consent was obtained by all participants in this study. Brooklyn Hospital Center IRB issued approval 1453304-1. Thank you for submitting the Publication materials for this project. The Brooklyn Hospital Center IRB has APPROVED your submission. No further action on submission 1453304-1 is required at this time. The following items are acknowledged in this submission: • Abstract/Summary Helminth • Application Form - Case Report Review Application • Consent Form. Conflicts of interest: In compliance with the ICMJE uniform disclosure form, all authors declare the following: Payment/services info: All authors have declared that no financial support was received from any organization for the submitted work. Financial relationships: All authors have declared that they have no financial relationships at present or within the previous three years with any organizations that might have an interest in the submitted work. Other relationships: All authors have declared that there are no other relationships or activities that could appear to have influenced the submitted work.

\section{References}

1. Copelan A, Bahoura L, Tardy F, Kirsch M, Sokhandon F, Kapoor B: Etiology, diagnosis, and management of bilomas: a current update. Tech Vasc Interv Radiol. 2015, 18:236-243. 10.1053/j.tvir.2015.07.007

2. Tana C, D'Alessandro P, Tartaro A, Tana M, Mezzetti A, Schiavone C: Sonographic assessment of a suspected biloma: a case report and review of literature. World J Radiol. 2013, 5:220-225. 10.4329/wjr.v5.i5.220

3. Zhang B, Guo Y, Wu K, Shan H: Intrahepatic biloma following transcatheter arterial chemoembolization for hepatocellular carcinoma: incidence, imaging features and management. Mol Clin Oncol. 2017, 6:937-943. 10.3892/mco.2017.1235

4. Peters JH, Ellison EC, Innes JT, et al.: Safety and efficacy of laparoscopic cholecystectomy: a prospective analysis of 100 initial patients. Ann Surg. 1991, 213:3-12. 10.1097/00000658-199101000-00002 


\section{Cureus}

5. Barone JE, Lincer RM: Correction: a prospective analysis of 1518 laparoscopic cholecystectomies . N Engl J Med. 1991, 325:1517-1518. 10.1056/nejm199111213252117

6. Chang IS, Rhim H, Kim SH, Kim YS, Choi D, Park Y, Lim HK: Biloma formation after radio-frequency ablation of hepatocellular carcinoma: incidence, imaging features, and clinical significance. Am J Roentgenol. 2010, 195:1131-1136. 10.2214/AJR.09.3946

7. Glenn F: Injuries to the liver and biliary tract. Am J Surg. 1956, 91:534-539. 10.1016/0002-9610(56)90282-3

8. Liu J, Wu Y, Xu E, et al.: Risk factors of intrahepatic biloma and secondary infection after thermal ablation for malignant hepatic tumors. Int J Hyperthermia. 2019, 36:979-984. 10.1080/02656736.2019.1660422

9. Würstle S, Göß A, Spinner CD, et al.: A retrospective clinical and microbial analysis of 32 patients with bilomas. BMC Gastroenterol. 2019, 19:50. 10.1186/s12876-019-0968-2

10. Gould L, Patel A: Ultrasound detection of extrahepatic encapsulated bile: "biloma" . Am J Roentgenol. 1979, 132(6):1014-15. 10.2214/ajr.132.6.1014

11. Thompson CM, Saad NE, Quazi RR, Darcy MD, Picus DD, Menias CO: Management of iatrogenic bile duct injuries: role of the interventional radiologist. Radiographics. 2013, 33:117-134. 10.1148/rg.331125044

12. Christoforidis E, Vasiliadis K, Goulimaris I, et al.: A single center experience in minimally invasive treatment of postcholecystectomy bile leak, complicated with biloma formation. J Surg Res. 2007, 141:171175. 10.1016/j.jss.2006.07.012 